\title{
Desplazamiento Posterior Bilateral del Disco de la Articulación Temporomandibular: Evidencia en Resonancia Magnética
}

\author{
Bilateral Posterior Displacement of the Temporomandibular \\ Joint Disc: Evidence on Magnetic Resonance Imaging
}

\author{
Germán Aguilar'; Adalsa Hernández-Andara²; Ana I. Ortega-Pertuz ${ }^{3}$ \& Maira Quevedo-Piña ${ }^{4}$
}

\begin{abstract}
AGUILAR, G.; HERNÁNDEZ-ANDARA, A.; ORTEGA-PERTUZ, A. I. \& QUEVEDO-PIÑA, M. Desplazamiento posterior bilateral del disco de la articulación temporomandibular: evidencia en resonancia magnética. Int. J. Odontostomat., 15(1):111$118,2021$.

RESUMEN: El desplazamiento del disco articular (DA) constituye una artropatía intracapsular en la que el disco se desplaza de su relación funcional con el cóndilo mandibular y la porción articular del hueso temporal. El desplazamiento del disco puede ser antero-medial, anterior, antero-lateral, lateral, medial o posterior, siendo este ultimo uno de los menos frecuentes. El presente reporte tuvo como objetivo describir las manifestaciones clínicas y evaluación imagenológica mediante resonancia magnética (RM) de un desplazamiento posterior del disco (DPD) en la articulación temporomandibular (ATM). Presentación del caso: paciente masculino, de 35 años de edad quien a la exploración clínica, refirió dolor a la palpación lateral y posterior en la ATM izquierda, se observó desvío mandibular hacia el lado derecho, dificultad para realizar movimientos de lateralidad y limitación de la apertura oral. Intraoralmente, se evidenció mordida cruzada en zona canina derecha y facetas de desgaste en caninos superiores e incisivos inferiores. En imágenes sagitales de RM a boca cerrada se verificó en ambas articulaciones, el aplanamiento del DA, la localización de la banda anterior sobre la vertiente anterior de la cabeza de la mandíbula, de manera que el resto del disco estaba extendido hacia la vertiente posterior, ocupando parte de la zona retrodiscal; en el plano coronal la posición del DA estaba íntimamente relacionada con los polos lateral y medial de la cabeza de la mandíbula. Las características imagenológicas fueron sugestivas de DPD. El DPD es una artropatía poco frecuente de la ATM, siendo que las imágenes por RM muestran con precisión la relación DA-cóndilo, lo que posibilita su diagnóstico y permite al clínico tomar las decisiones idóneas para la atención del paciente.
\end{abstract}

PALABRAS CLAVE: articulación temporomadibular, disfunción de la articulación temporomandibular, resonancia magnética.

\section{INTRODUCCIÓN}

Las artropatías de la articulación temporomandibular (ATM) son alteraciones músculo esqueléticas comunes que pueden provocar dolor y discapacidad (Afroz et al., 2018). A menudo la disfunción temporomandibular está relacionada con alteraciones del complejo disco-cóndilo, donde se observan cambios en la relación fisiológica del disco articular (DA) con respecto a la cabeza de la mandíbula, el tubérculo articular y la fosa mandibular del hueso temporal (González-García, 2019).
EI DA está formado por tejido conectivo denso sin inervación ni vascularización. Desde el plano sagital se observa bicóncavo con una banda posterior gruesa, una zona intermedia delgada y una banda anterior más delgada que la posterior, estableciendo una relación media de 3:1:2 (Isberg, 2006). Cuando la mandíbula se encuentra en reposo, la banda intermedia del DA se localiza entre la vertiente anterior de la cabeza de la mandíbula y la vertiente posterior del tubérculo articular, de manera que la banda posterior se sitúa

\footnotetext{
${ }^{1}$ Facultad de Odontología, Universidad de Antioquia, Medellín,Colombia.

2 Unidad de Imagen Dentomaxilofacial, ClínicaFélix Boada, Caracas, Venezuela.

${ }^{3}$ Instituto de Investigaciones, Facultad de Odontología, Universidad del Zulia, Maracaibo, Estado Zulia, Venezuela.

${ }^{4}$ Facultad de Odontología. Universidad de Carabobo. Valencia, Estado Carabobo, Venezuela.
} 
más o menos próxima a la posición de 12 horas en relación con la fosa mandibular y la banda anterior se coloca por delante del cóndilo (Isberg, 2011). EI DA divide la articulación en dos compartimientos superior o disco-temporal e inferior o disco-mandibular (Behzadi et al., 2020). Los compartimientos articulares presentan los recesos anterior y posterior revestidos de líquido sinovial que proporciona lubricación para la articulación, el espacio posterior al disco y el cóndilo se denomina "tejido retrodiscal" o "zona bilaminar" muy rica en vasos y terminaciones nerviosas (Tamimi et al., 2019).

Las alteraciones en el desplazamiento del disco conocidas como artropatías o alteraciones intracapsulares, han sido consideradas como un mecanismo subyacente en la patogénesis de la disfunción de la ATM. El desplazamiento del disco se ha definido como una disrupción de las relaciones internas de la ATM en la cual el DA se desplaza de su relación funcional con el cóndilo mandibular y la porción articular del hueso temporal y esta modificación se asocia con síntomas clínicos como dolor, sonidos articulares y disfunción mandíbular (Kumar et al., 2015).

El $70 \%$ de alteraciones intracapsulares se han relacionado con mal posición del DA. Las principales teorías de estas alteraciones, así como de los procesos degenerativos, incluyen tensión mecánica desequilibrada, compresión anormal, sobrecarga funcional, aumento de la fricción articular y microtrauma (Behzadi et al.). El desplazamiento del disco es la anormalidad más común observada en imágenes por Resonancia magnética (RM) de la ATM. Con mayor frecuencia el desplazamiento es antero-medial, anterior y anterolateral. El desplazamiento posterior de disco (DPD) se ha considerado extremadamente raro (Chossegros et al., 2001; Melis et al., 2011) debido a que la orientación oblicua del músculo pterigoideo lateral y la angulación del cóndilo tienden a dirigir la mayoría de los desplazamientos discales en una trayectoria anteromedial (Molinari et al., 2007). También se ha sugerido que la mayoría de los DPD son resultados de problemas de adhesión en el espacio superior de la ATM (Kim et al., 2016).

En el DPD la banda posterior está en contacto aparente con la zona bilaminar y la banda anterior está en la posición entre las 2:00 a 3:00 en punto (Chalkoo et al., 2017). Para Tomas et al. (2006) en el DPD la banda posterior está desplazada más allá de la posición de la una en punto. Okesson refiere que cuando existe adhesión en el compartimiento superior (entre el disco y la fosa mandibular) y se presenta alargamiento de los ligamentos colateral y anterior, la cabeza de la mandíbula se mueve hacia adelante y el disco no lo acompaña en el recorrido y queda en posición posterior (Kim et al.).

Westesson et al. (1998) han clasificado los DPD en tres categorías, caracterizadas de la siguiente manera:en la primera, una delgada banda de tejido discal se ubica en la cabeza de la mandíbula y se extiende en una posición posterior; en la segunda el disco está completamente desplazado posteriormente y para la tercera, el disco está perforado con una porción del mismo desplazada posteriormente. Aunque no hay consenso general sobre la clasificación de DPD en la ATM, se ha utilizado la terminología DPD con reducción y DPD sin reducción (Kim et al.).

La morfología del DA en condiciones normales es bicóncava. Para evaluar su forma Murakami et al., establecieron una clasificación de acuerdo a la forma observada en el plano sagital: bicóncava, cuando las superficies superiores e inferiores son cóncavas, biplanar en donde el espesor del disco es uniforme; biconvexo, ambas superficies superior e inferior son convexas; hemiconvexa, la superficie superior es cóncava y la inferior es convexa y doblado, el disco está doblado en su parte central (de Farias et al., 2015).

Los síntomas manifestados por los pacientes con DPD incluyen dolor, imposibilidad para el cierre oral en máxima intercuspidación (Huddleston Slater et al., 2005), desviación de la mandíbula hacia el lado afectado y limitación de apertura (Afroz et al., 2018). En este sentido, Okochi et al. (2008) reportaron que los pacientes con DPD de su estudio, mostraron una prevalencia de clicks en un $42 \%$, dolor $(26 \%)$, luxación de ATM (24\%), mordida abierta (21\%) y crepitación $(2 \%)$.

La prevalencia de DPD es baja, Tasaki et al. (1996) informaron que en 486 ATM estudiadas, solo tres casos $(0,6 \%)$ de los desplazamientos de disco fueron posteriores. Kumar et al. observaron que solo hubo un DPD representando el $2,3 \%$ en 44 articulaciones evaluadas Westesson et al., examinaron las imágenes de resonancia magnético (RM) de 3.200 pacientes con o sin síntomas clínicos en la ATM, y encontraron DPD en 23 pacientes, lo que representa menos del $1 \%$.

La RM se considera el gold standard para el diagnóstico de anomalías de la ATM, debido a que 
permite evaluar los tejidos blandos relacionados con la articulación, en particular, la relación disco-cóndilo y determinar el desplazamiento del DA (Okochi et al.; Ferreira et al., 2016; Bedran et al., 2019), sin exposición a radiación ionizante, ni procedimientos quirúrgicos invasivos (Nickel et al., 2018). En las imágenes de RM, la intensidad de señal relativamente baja del DA en la ATM normal, permite distinguirlo de los tejidos blandos circundantes debido a que se observa hipointenso. En general, el DA en presencia de artropatía, tiene una intensidad de señal notablemente menor que el disco normal (de Oliveira Amaral et al., 2013). Esta especificidad ha hecho que los clínicos se apoyen en las imágenes de la RM., además de proporcionar imágenes estáticas de buena calidad, permite analizar la posición del disco y el movimiento condilar durante todo el proceso dinámico de apertura y cierre oral mediante la obtención de múltiples imágenes en serie (Bedran et al.).

En consideración a estas ventajas de RM con un $95 \%$ de precisión en la evaluación de la posición y forma del disco y $93 \%$ en evaluación de cambios óseos, se erige como el método de imagen a elección en las artropatías de la ATM (Kumar et al.). Debido a que la intensidad de la señal del hueso, el cartílago, el DA, los músculos masticatorios y el líquido sinovial difieren, la estructura de la ATM puede mostrarse claramente. El protocolo para el estudio de la ATM incluye secuencias de pulso específicas, las cuales se han ido modificando para una mejor evaluación de las estructuras, entre las que se conocen: densidad protónica (DP) que corresponde al número de protones en el volumen estudiado. Está indicada para determinar la posición del disco, morfología discal, efusión y cambios óseos. T2, en esta secuencia se observa el líquido con una señal de alta intensidad, por lo que es útil en la identificación de lesiones patológicas que suelen caracterizarse por un aumento en el contenido de agua (Lamot et al., 2013). T2*, son las secuencias potenciadas en T2 para la detección de efusión, que se manifiesta como

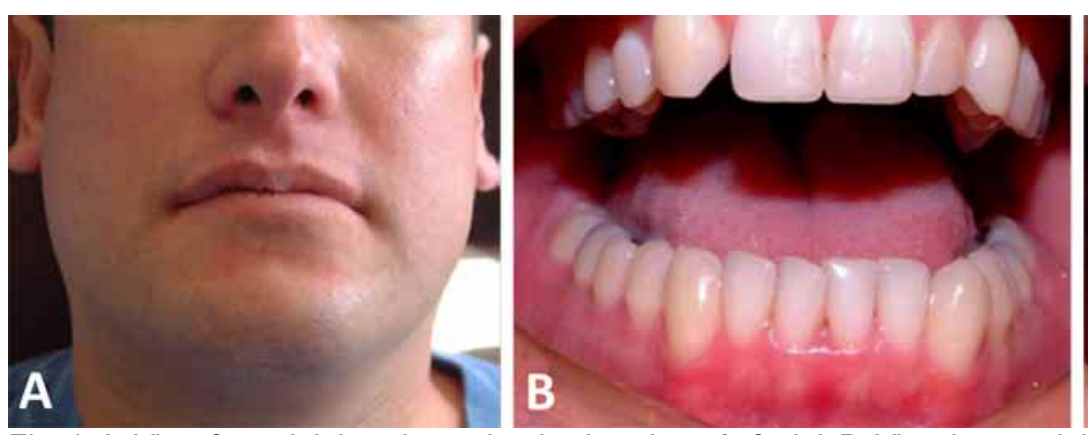

Fig. 1. A. Vista frontal del paciente donde sin asimetría facial. B. Vista intraoral donde se evidencia una apertura oral limitada y C. Vista intraoral en oclusión obsérvese mordida cruzada canina izquierda. áreas de hiperintensidad. El tiempo de excitación (TE) o tiempo de emisión del pulso de radiofrecuencia y el tiempo de relajación (TR) o tiempo de recepción de la señal, se miden en milisegundos y su duración depende de la potenciación que se desea obtener ya que todos los tejidos no liberan energía a la misma intensidad (Costa Subías \& Jerez, 2015). La secuencia dinámica documenta la posición del disco y su relación con la cabeza de la mandíbula a fin de determinar si se produce o no reducción en el desplazamiento del disco (De La Hoz Polo \& Navallas et al., 2014).

Tomando en consideración lo anteriormente expuesto, el objetivo de este reporte es presentar el caso de un paciente de sexo masculino con manifestaciones clínicas y evaluación imagenológica por RM, cuyo diagnóstico definitivo fue DPD parcial bilateral, se exponen los hallazgos y se contrasta con las investigaciones referentes a esta condición.

\section{PRESENTACIÓN DEL CASO}

Paciente masculino de 35 años de edad, referido por su odontólogo tratante para realizar estudios de RM en la ATM por indicar durante la anamnesis, sintomatología dolorosa con un tiempo de evolución de dos días, dificultad y limitación para la masticación. A la exploración física extraoral manifiesta dolor a la palpación lateral y posterior en ATM izquierda, desviación mandibular hacia el lado derecho, dificultad para realizar movimientos mandibulares laterales y una apertura oral máxima de $20 \mathrm{~mm}$. En cuanto a los antecedentes odontológicos, refiere haber utilizado dispositivo interoclusal para el tratamiento de bruxismo. Al examen clínico intraoral se observómordida cruzada en zona canina derecha, facetas de desgaste en cúspides de caninos superiores y margen incisal de incisivos inferiores, así como restauraciones de amalgama en 1.6, 2.6, 3.6 y 4.6 en buen estado (Fig. 1). 
Las imágenes de RM de ambas ATM se obtuvieron en un equipo con un campo magnético de 1.5 Tesla (Diamond Select Achieva, Phillips, Eindhoven, Netherlands), utilizando bobinas duales específicas para ATM. Con el paciente en posición supina se adquirieron imágenes de $3 \mathrm{~mm}$ de espesor en el plano sagital oblicuo, perpendicular al eje longitudinal del cóndilo en sentido anteroposterior y el plano coronal oblicuo, paralelo al eje cóndilo lateromedial. Para cada ATM, en boca abierta y boca cerrada en el plano sagital oblicuo, se aplicó el siguiente protocolo: DP (TE 20.0 / TR 1565.0), y en T2 (TE 150.0 / TR 1800.0). Para coronal corregida en boca cerrada se adquirieron imágenes en DP (TE 20.0 / TR 1565.0). En la secuencia dinámica se realizaron imágenes en varios grados de apertura oral desde una posición cerrada hasta boca abierta. Las imágenes seriadas fueron posteriormente reconstruidas en T2* (TE 20.0 / TR 80.0). Las imágenes fueron almacenadas en formato DICOM.

En el plano sagital oblicuo boca cerrada, se observó imagen hipointensa correspondiente al DA aplanado, tanto en ATM derecha como izquierda, la banda anterior se encontraba localizada sobre la vertiente anterior de la cabeza de la mandíbula y el resto del disco se extendía hacia la vertiente posterior, ocupando parte de la zona retrodiscal (Fig. 2). En el plano coronal, boca cerrada, la posición del DA estaba íntimamente relacionada con los polos lateral y medialen ambas ATM. Las características imagenológicas fueron sugestivas de DPD en ambas ATM (Fig. 3).
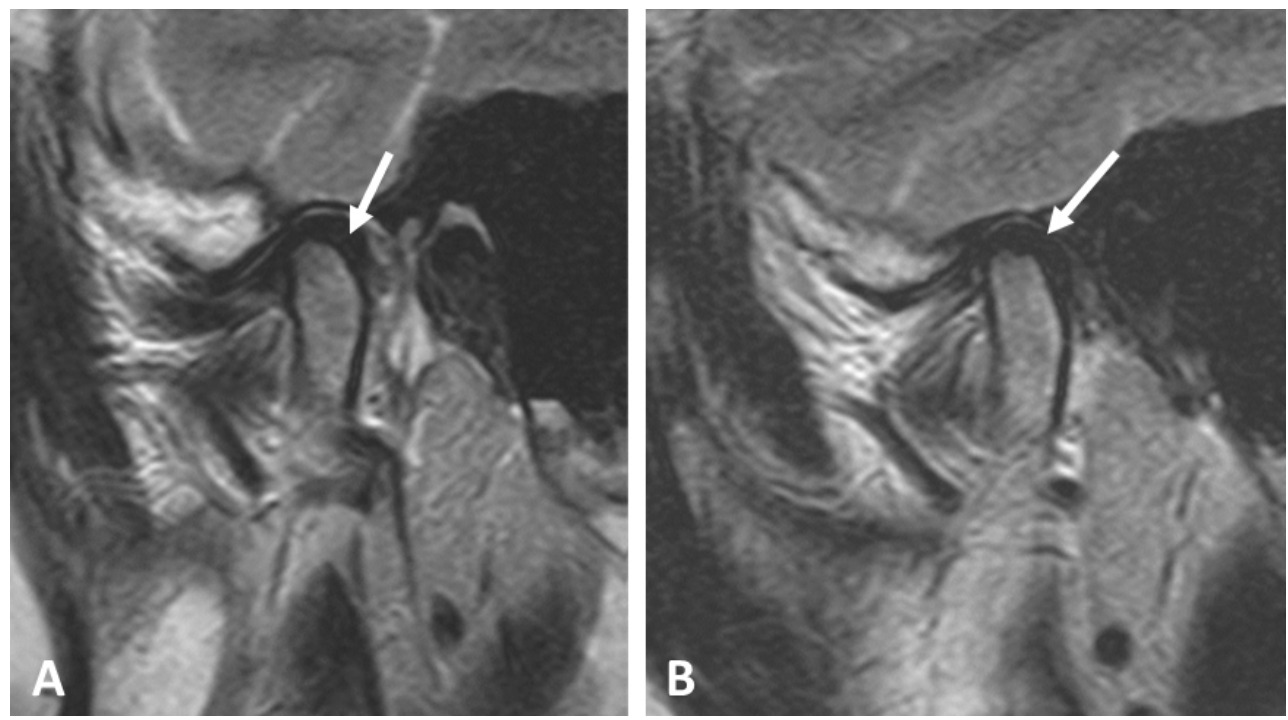

Fig. 2. Vista sagital de imágenes de resonancia magnética ponderadas en T2 boca cerrada. A. Lado derecho y $\mathrm{B}$. Lado izquierdo. Las flechas señalan el disco articular con desplazamiento posterior parcial en ambas articulaciones temporomandibulares.
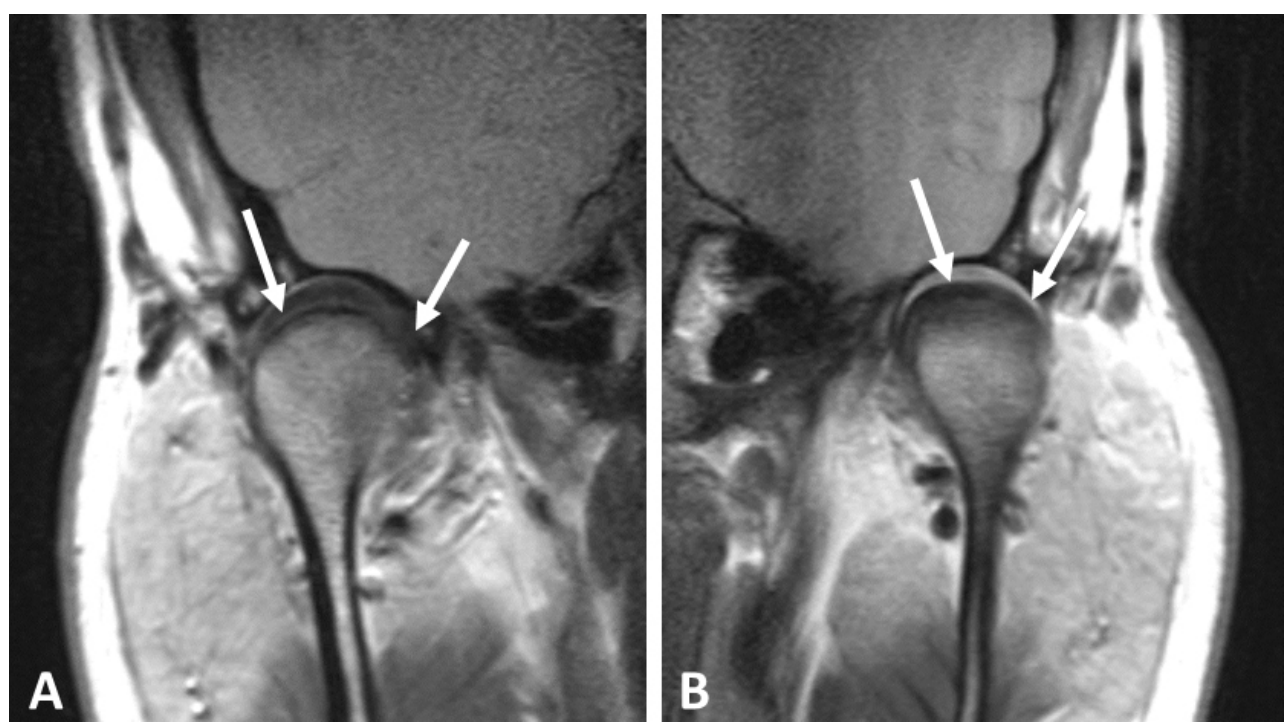

Fig. 3. Vista coronal de imágenes de resonancia magnética ponderadas en DP. A. Lado derecho y B. Lado izquierdo. Las flechas señalan el disco articular unido a los polos lateral y medial del cóndilo en ambas articulaciones temporomandibulares. 
Al evaluar las imágenes dinámicas, adquiridas en cortes seriados y luego reconstruidos, se observó que el DA, para ATM derecha e izquierda, permanece en contacto con la cabeza de la mandíbula desde el inicio de la apertura oral hasta la máxima excursión condilar (Figs. 4 y 5 ).
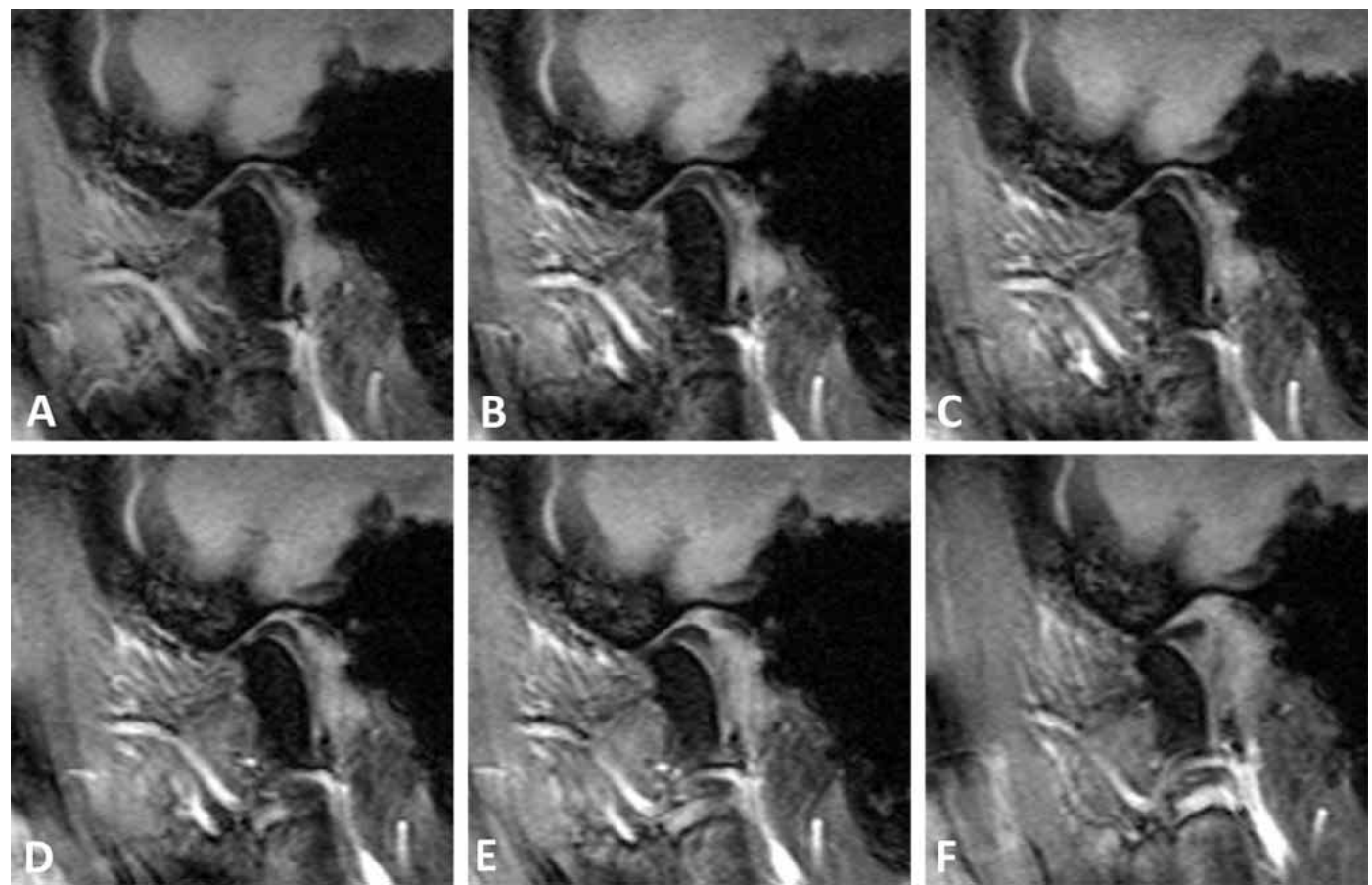

Fig. 4. En articulación temporomandibular del lado derecho se observa en secuencia dinámica de imágenes de resonancia magnética adquiridas en $\mathrm{T} 2^{*}$, la posición posterior parcial del disco articular durante la translación del proceso condilar.
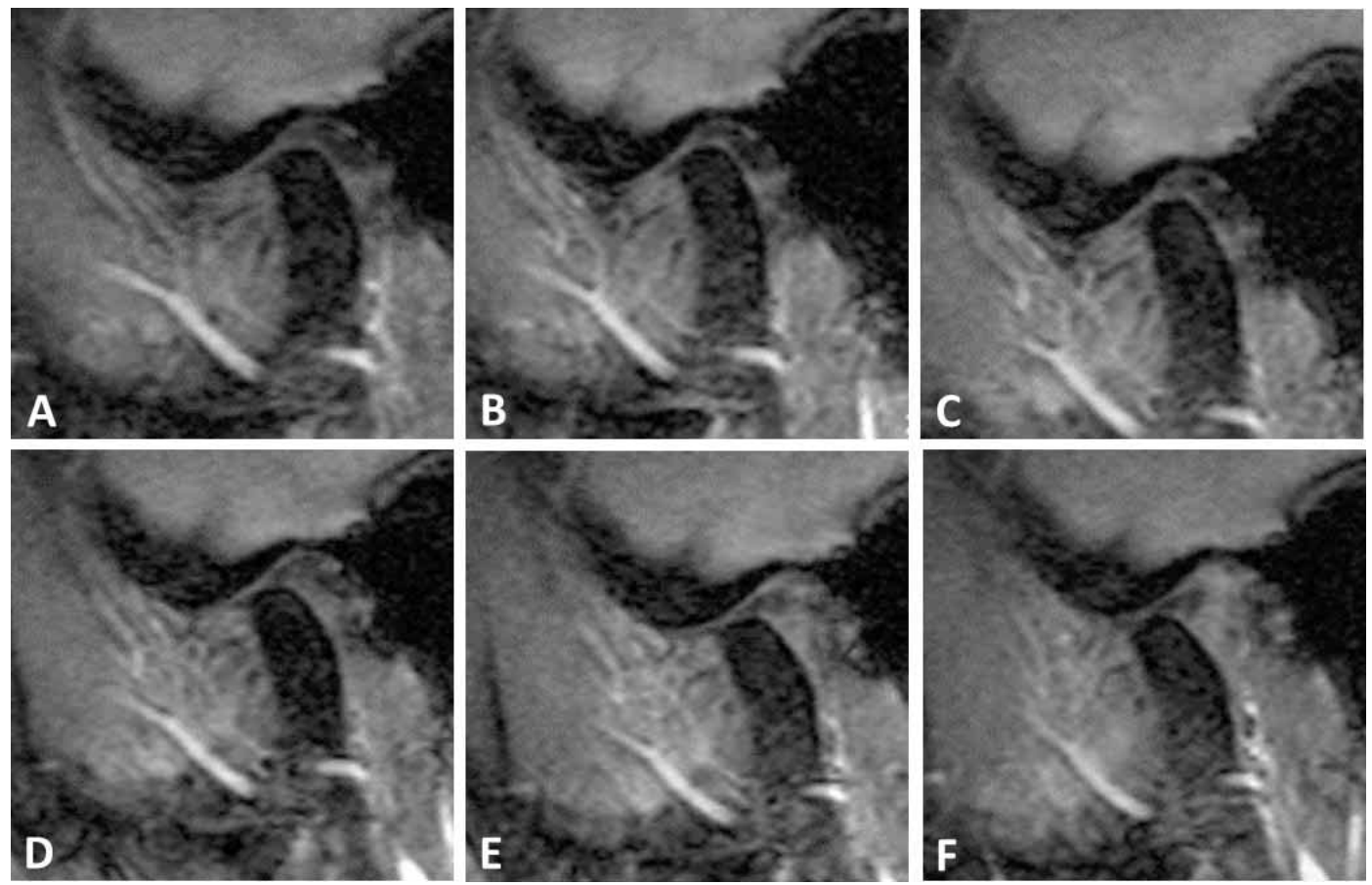

Fig. 5. En articulación temporomandibular del lado izquierdo se observa en secuencia dinámica de imágenes de resonancia magnética adquiridas en $\mathrm{T} 2^{*}$, la posición posterior parcial del disco articular durante la translación del proceso condilar. 


\section{DISCUSIÓN}

La ATM es una de las articulaciones más complejas del cuerpo humano, debido a su movimiento único, combinando rotacióny translación. EI DA juega un papel importante para mantener el funcionamiento armónico de la ATMy su posición adecuada permite un movimiento de bisagra, así como acciones de deslizamiento entre el tubérculo articular del temporal y la cabeza de la mandíbula. EI DPD es considerado poco frecuente, en tal sentido, Afroz et al. (2018) en su investigación concluyen que no hay conocimiento detallado de la prevalencia, factores de riesgo o características clínicas de esta entidad.

En relación a la sintomatología de los pacientes con DPD, el dolor es un elemento común en varias investigaciones (Chossegros et al.; Okochi et al.; Chiba et al., 2007), aunque también se presentan casos donde los pacientes no manifiestan sintomatología dolorosa (Chossegros et al.; Kim et al.). En las investigaciones consultadas, algunos pacientespueden ubicar la zona comprometida en la ATM derecha o izquierda (Kim et al.), otros mencionan ambas ATM, o bien que la sintomatología dolorosa se presenta a la palpación en la región de la ATM, específicamente en el músculo masetero y/o al realizar movimientos de lateralidad y protusiva (Afroz et al., 2020). En este reporte, el paciente manifiesta dolor a la palpación lateral y posterior, localizado a nivel de la ATM izquierda. El dolor también se ha asociado a la morfología del disco. Al respecto, Kim et al. informaron que en el disco aplanado está presente en el $19 \%$ de los casos, coincidente con la forma del disco observado en el paciente a la evaluación con RM en el plano sagital.

Por su parte los estudios informan la presencia de desoclusión en el sector posterior en el lado afectado que suele ser aproximadamente de $1 \mathrm{~mm}$ (Young, 2015) y la incapacidad repentina de realizar una máxima intercuspidación, siendo que el paciente manifiesta sentir la presencia de material elástico entre los molares (Tomas et al.; Westesson et al.; Huddleston Slater et al.) y restricción de la apertura oral. Estos hallazgos fueron coincidentes en el caso referido, quien manifestó dificultad para la masticación y limitación de apertura $(20 \mathrm{~mm})$.

El bruxismo se asocia con contractura muscular local crónica, inflamación e hipertrofia muscular localizada, que a su vez puede causar dolor miofacial (Garip et al., 2018). También se ha informado que el bruxismo es una posible causa de alteraciones del disco, ya que la sobrecarga compresiva puede alterar el tejido conectivo de la ATM. El bruxismo también se relaciona frecuentemente con diversas alteraciones temporomandibulares. Sin embargo, aún no se ha demostrado una asociación causal entre este comportamiento no fisiológico y las alteraciones del disco (Molinari et al.). En el presente caso, el paciente comunicó en la anamnesis que había utilizado aparatología para el bruxismo y se identificaron facetas de desgaste al examen intraoral.

La RM de la ATM juega un papel importante en el diagnóstico de trastorno interno porque permite la visualización directa del DA en apertura y cierre oral, proporcionando información sobre la ubicación, morfología, intensidad de señal y estructura del DA (Kumar et al.). Las imágenes se obtienen en múltiples planos, aumentando la precisión del diagnóstico sin necesidad de utilizar material de contraste y permite evaluar los tejidos blandos adyacentes (Tomas et al.; de Oliveira Amaral et al.).

Los cambios morfológicos en el DA son de interés clínico porque son signos de patología y disfunción de ATM (de Farias et al.; de Oliveira Amaral et al.). La configuración bicóncava parece cambiar como resultado del desplazamiento de disco de su posición normal. La forma puede verse influenciada por diversos factores, como una función anormal o composición del disco en sí. La etiología de las alteraciones en el disco sigue siendo controvertida (Chang et al., 2018). La deformación del DA implica porciones progresivamente diferentes del mismo y se presume es el resultado de una adaptación estructural dinámica que extiende la tensión mecánica sobre superficies más grandes (Molinari et al.), este proceso ocasiona la pérdida de su morfología original, observándose en el caso presentado un DA aplanado en ambas articulaciones. En la investigación de Bedran et al. el DPD fue significativamente asociado con la deformidad del DA. El aplanamiento del DA también representa un hallazgo patológico, en las imágenes de RM se observa como la intensidad de la señal del DA que normalmente es de intermedia a alta, disminuye (Tomas et al.). Esta disminución de la señal coincide con lo observado en la RM del pacienteen el plano sagital en T2.

Se ha sugerido que las imágenes sagitales y coronales de RM permiten diagnosticar todos los tipos de desplazamientos de DA. En la vista coronal puede identificarse el desplazamiento lateral y medial. 
Las secuencias axiales y coronales también son de utilidad para evaluar la morfología de la cabeza de la mandíbula (de Farias et al.). En el estudio de Farias et al., se presentaron dos casos con DPD, en uno de ellos la forma del cóndilo era redondeada y en el otro se observó una morfología angulada, situación coincidente con el presente caso, donde la cabeza de la mandíbula tenía forma redondeada. Los casos clínicos reportados en la literatura informan DPD unilateral (Chossegros et al.; Kim et al.; Huddleston Slater et al.; Afroz et al., 2020; Chiba et al.). Únicamente el estudio de Westesson et al., menciona que de 21 pacientes con imágenes de RM y artrografía, nueve de ellos presentaban DPD bilateral. En el caso presentado el DPD se observó en ambas ATM, sobre este hallazgo la casuística es escasa por tanto no se podría establecer una prevalencia sobre la presencia de DPD unilateral o bilateral.

Por otro lado, la secuencia RM ponderada en T2 es un método no invasivo en comparación con la RM con material de contraste, ésta permite detectar la presencia de un proceso inflamatorio sinovial en la ATM (Suenaga et al., 2016). En el protocolo utilizado en este reporte se utilizó T2* y DP y no se observó efusión en los espacios articulares.

La RM dinámica complementa la RM estática al proporcionar información adicional sobre la movilidad del DA y la cabeza de la mandíbula, el desplazamiento del mismo y los cambios topográficos en la relación DA-cabeza de la mandíbula durante varias etapas del movimiento de apertura oral (Shimazaki et al., 2007). Las imágenes seriadas en este caso permitieron observar que el desplazamiento del DA fue parcial, es decir se mantuvo unido a la cabeza de la mandíbula en la trayectoria de apertura oral, como se observó en las imágenes de RM.

\section{CONCLUSIÓN}

El desplazamiento posterior de disco articular es una artropatía poco frecuente de la ATM. Entre los signos y síntomas están: dolor, limitación de apertura oral, dificultad para la masticación e imposibilidad para máxima intercuspidación del lado afectado. Para su evaluación, la RM se erige como el método de diagnóstico por imagen indicado para estudiar la relación DA - cabeza de la mandíbula. El diagnóstico preciso permitirá queel clínico tome las decisiones idóneas para la atención del paciente.
AGUILAR, G.; HERNÁNDEZ-ANDARA, A.; ORTEGAPERTUZ, A. I. \& QUEVEDO-PIÑA, M. Bilateral posterior displacement of the temporomandibular joint disc: evidence on magnetic resonance imaging. Int. J. Odontostomat., 15(1):111-118, 2021.

ABSTRACT: The displacement of the articular disc (AD) constitutes an intracapsular arthropathy in which the disc is displaced from its functional relationship with the mandibular condyle and the articular portion of the temporal bone. It is frequently medial, anteromedial, or anterolateral, with posterior displacement being rare. The objective of this report was to describe the clinical manifestations and magnetic resonance imaging (MRI) of a disc posterior displacement (PDD) in the temporomandibular joint (TMJ). Case presentation: a 35-year-old male patient who reported on clinical examination, pain on lateral and posterior palpation in the left TMJ; mandibular deviation to the right side, difficulty in making lateral movements, and limitation of mouth opening were observed. Intraorally, cross bite was observed in the right canine area and wear facets in the upper canines and lower incisors. In closed mouth MRI sagittal views, the flattening of the DA, the location of the anterior band on the anterior aspect of the mandibular head, was verified in both joints, so that the rest of the disc was extended towards the posterior aspect, occupying part from the retrodiscal zone; in the coronal plane the position of the DA was closely related to the lateral and medial poles of condyle. Imaging characteristics were suggestive of PDD. Conclusions: PDD is a rare arthropathy of the TMJ, since MRI images accurately show the DA-condyle relationship, which enables its diagnosis and allows the clinician to make the best decisions for patient care.

KEY WORDS: temporomandibular joint, temporomandibular joint disorder, magnetic resonance imaging.

\section{REFERENCIAS BIBLIOGRÁFICAS}

Afroz, S.; Naritani, M.; Hosoki, H. \& Matsuka, Y. Posterior disc displacement of the temporomandibular joint: A rare case report. Cranio, 38(4):273-8, 2020

Afroz, S.; Naritani, M.; Hosoki, H.; Takechi, K.; Okayama, Y. \& Matsuka, Y. Prevalence of posterior disc displacement of the temporomandibular joint in patients with temporomandibular disorders: systematic review and meta-analyses. J. Oral Facial Pain Headache, 32(3):277-86, 2018.

Bedran, L. M. \& dos Santos, A. A. S. M. D. Changes in temporomandibular joint anatomy, changes in condylar translation, and their relationship with disc displacement: magnetic resonance imaging study. Radiol. Bras., 52(2):85-91, 2019.

Behzadi, F.; Mandell, J. C.; Smith, S. E. \& Guenette, J. P. Temporomandibular joint imaging: current clinical applications, biochemical comparison with the intervertebral disc and knee meniscus, and opportunities for advancement. Skeletal Radiol., 49(8):1183-93, 2020. 
Chalkoo, A. H.; Bhat, Z. A. \& Maqbool, A. MRI characteristics of disc displacement of temporomandibular disorders and its correlation with clinical findings in symptomatic and asymptomatic subjects. Int. J. Appl. Dent. Sci., 3(2):80-5, 2017.

Chang, C. L.; Wang, D. H.; Yang, M. C.; Hsu, W. E. \& Hsu, M. L. Functional disorders of the temporomandibular joints: Internal derangement of the temporomandibular joint. Kaohsiung J. Med. Sci., 34(4):223-30, 2018.

Chiba, M.; Watanabe, N. \& Echigo, S. Longitudinal MRI follow-up of non-reducible posterior disc displacement accompanied by bone marrow oedema in the mandibular condyle. Dentomaxillofacial Radiol., 36(5):304-7, 2007.

Chossegros, C.; Cheynet, F.; Guyot, L.; Bellot-Samson, V. \& Blanc, J. L. Posterior disk displacement of the TMJ: MRI evidence in two cases. Cranio, 19(4):289-93, 2001.

Costa Subías, J. \& Jerez, S. Resonancia Magnética Dirigida a Técnicos Superiores en Imagen Para El Diagnóstico. Madrid, Elsevier, 2015

de Farias, J. F. G.; Melo, S. L. S.; Bento, P. M.; Oliveira, L. S. A. F.; Campos, P. S. F. \& de Melo, D. P. Correlation between temporomandibular joint morphology and disc displacement by MRI. Dentomaxillofac. Radiol., 44(7):20150023, 2015.

De La Hoz Polo, M. \& Navallas, M. La articulación temporomandibular en la artritis idiopática juvenil: lo que el radiólogo debe buscar en la resonancia magnética. Radiología, 56(5):440-50, 2014.

de Oliveira Amaral, R.; de Lima Damasceno, N. N.; de Souza, L. A. \& Devito, K. L. Magnetic resonance images of patients with temporomandibular disorders: Prevalence and correlation between disk morphology and displacement. Eur. J. Radiol., 82(6):990-4, 2013.

Ferreira, L. A.; Grossmann, E.; Januzzi, E.; de Paula, M. V. Q. \& Carvalho, A. C. P. Diagnosis of temporomandibular joint disorders: indication of imaging exams. Braz. J. Otorhinolaryngol., 82(3):341-52, 2016.

Garip, H.; Tufekcioglu, S. \& Kaya, E. Changes in the temporomandibular joint disc and temporal and masseter muscles secondary to bruxism in Turkish patients. Saudi Med. J., 39(1):81-5, 2018.

González-García, R. Breves consideraciones sobre el síndrome de dolor-disfunción de la atm en el paciente con deformidad dentofacial. Rev. Esp. Cir. Oral Maxilofac., 41(4):157-9, 2019.

Huddleston Slater, J. J. R.; Lobbezoo, F.; Hofman, N. \& Naeije, M. Case report of a posterior disc displacement without and with reduction. J. Orofac. Pain, 19(4):337-42, 2005.

Isberg, A. Disfunción de la Articulación Temporomandibular. Una Guía Práctica para el Profesional. São Paulo, Artes médicas Latinoaméricas, 2006.

Isberg, A. Temporomandibular Joint Dysfunction. A Practitioner's Guide. Estocolmo, MosebakeTorg, 2011.

Kim, J.; Kim, M. J. \& Kho, H. S. Posterior disk displacement in the temporomandibular joint: a report of two cases. J. Oral Med. Pain, 41(3):137-43, 2016.

Kumar, R.; Pallagatti, S.; Sheikh, S.; Mittal, A.; Gupta, D. \& Gupta, $S$. Correlation between clinical findings of temporomandibular disorders and MRI characteristics of disc displacement. Open Dent. J.., 9:273-81, 2015.

Lamot, U.; Strojan, P. \& Popovič, K. S. Magnetic resonance imaging of temporomandibular joint dysfunction-correlation with clinical symptoms, age, and gender. Oral Sur. Oral Med. Oral Pathol. Oral Radiol., 116(2):258-63, 2013.

Melis, M.; Di Giosia, M. \& Secci, S. Temporomandibular joint disk fracture: a case report. Cranio, 29(3):227-31, 2011.

Molinari, F.; Manicone, P. F.; Raffaelli, L.; Raffaelli, R.; Pirronti, T. \& Bonomo, L. Temporomandibular joint soft-tissue pathology, I: Disc abnormalities. Semin. Ultrasound CT MR, 28(3):192204, 2007.
Nickel, J.C.; Iwasaki, L. R.; Gonzalez, Y. M.; Gallo, L. M. \& Yao, H. Mechanobehavior and ontogenesis of the temporomandibular joint. J. Dent. Res., 97(11):1185- 92, 2018.

Okochi, K.; Ida, M.; Honda, E.; Kobayashi, K. \& Kurabayashi, T. $\mathrm{MRI}$ and clinical findings of posterior disk displacement in the temporomandibular joint. Oral Surg. Oral Med. Oral Pathol. Oral Radiol. Endod., 105(5):644-8, 2008.

Shimazaki, Y.; Saito, K.; Matsukawa, S.; Onizawa, R.; Kotake, F.; Nishio, R. \& Abe, K. Image quality using dynamic MR imaging of the temporomandibular joint with true-FISP sequence. Magn. Reson. Med. Sci., 6(1):15-20, 2007.

Suenaga, S.; Nagayama, K.; Nagasawa, T.; Indo, H. \& Majima, H. J. The usefulness of diagnostic imaging for the assessment of pain symptoms in temporomandibular disorders. Jpn. Dent. Sci. Rev., 52(4):93-106, 2016.

Tamimi, D.; Kocasarac, H. D. \& Mardini, S. Imaging of the temporomandibular joint. Semin. Roentgenol., 54(3):282-301, 2019.

Tasaki, M. M.; Westesson, P. L.; Isberg, A. M.; Ren, Y. F. \& Tallents, R. H. Classification and prevalence of temporomandibular joint disk displacement in patients and symptom-free volunteers. Am. J. Orthod. Dentofacial Orthop., 109(3):249-62, 1996.

Tomas, X.; Pomes, J.; Berenguer, J.; Quinto, L.; Nicolau, C.; Mercader, J. M. \& Castro, V. MR imaging of temporomandibular joint dysfunction: a pictorial review. Radiographics, 26(3):765-81, 2006.

Westesson, P. L.; Larheim, T. A. \& Tanaka, H. Posterior disc displacement in the temporomandibular joint. J. Oral Maxillofac. Surg., 56(11):1266-73, 1998.

Young, A. L. Internal derangements of the temporomandibular joint: A review of the anatomy, diagnosis, and management. J. Indian Prosthodont. Soc., 15(1):2-7, 2015

Dirección para correspondencia:

Dra. Adalsa Hernández-Andara

Unidad de Diagnóstico por Imagen

Clínica Félix Boada

Calle Bolívar, Baruta

Caracas

VENEZUELA

Email: adasa1@yahoo.com 